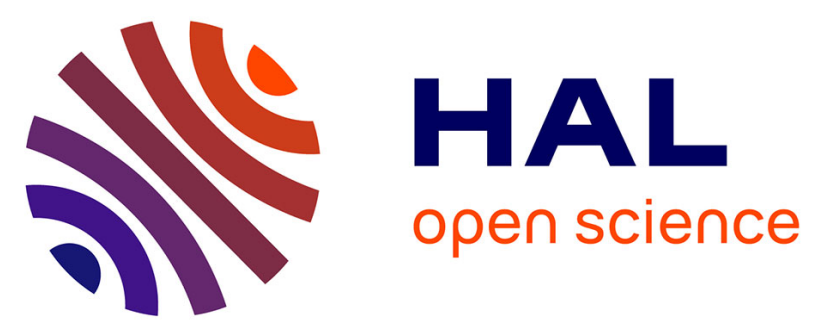

\title{
Theoretical model of ice nucleation induced by inertial acoustic cavitation. Part 2: Number of ice nuclei generated by a single bubble
}

C. Cogne, S. Labouret, R. Peczalski, Olivier Louisnard, Fabien Baillon, Fabienne Espitalier

\section{To cite this version:}

C. Cogne, S. Labouret, R. Peczalski, Olivier Louisnard, Fabien Baillon, et al.. Theoretical model of ice nucleation induced by inertial acoustic cavitation. Part 2: Number of ice nuclei generated by a single bubble. Ultrasonics Sonochemistry, 2016, 28, p. 185-191. 10.1016/j.ultsonch.2015.07.019 . hal-01620296

\section{HAL Id: hal-01620296 \\ https://hal.science/hal-01620296}

Submitted on 5 Sep 2018

HAL is a multi-disciplinary open access archive for the deposit and dissemination of scientific research documents, whether they are published or not. The documents may come from teaching and research institutions in France or abroad, or from public or private research centers.
L'archive ouverte pluridisciplinaire HAL, est destinée au dépôt et à la diffusion de documents scientifiques de niveau recherche, publiés ou non, émanant des établissements d'enseignement et de recherche français ou étrangers, des laboratoires publics ou privés. 


\title{
Theoretical model of ice nucleation induced by inertial acoustic cavitation. Part 2: Number of ice nuclei generated by a single bubble
}

\author{
C. Cogné ${ }^{\mathrm{a}}$, S. Labouret ${ }^{\mathrm{a}}$, R. Peczalski ${ }^{\mathrm{a}, *}$, O. Louisnard $^{\mathrm{b}}$, F. Baillon ${ }^{\mathrm{b}}$, F. Espitalier ${ }^{\mathrm{b}}$ \\ ${ }^{a}$ University of Lyon, France, Université Claude Bernard Lyon 1, Laboratoire d'Automatique et de Génie des Procédés (LAGEP UMR CNRS 5007), Campus de la Doua, Bât. CPE, \\ 69616 Villeurbanne, France \\ ${ }^{\mathrm{b}}$ University of Toulouse, France, Ecole Nationale Supérieure des Mines d'Albi-Carmaux, Centre de Recherche d'Albi en génie des Procédés des Solides Divisés, de l'Énergie et \\ de l'Environnement (RAPSODEE UMR CNRS 5302), Campus Jarlard, 81013 Albi, France
}

\begin{abstract}
A B S T R A C T
In the preceding paper (part 1), the pressure and temperature fields close to a bubble undergoing inertial acoustic cavitation were presented. It was shown that extremely high liquid water pressures but quite moderate temperatures were attained near the bubble wall just after the collapse providing the necessary conditions for ice nucleation. In this paper (part 2), the nucleation rate and the nuclei number generated by a single collapsing bubble were determined. The calculations were performed for different driving acoustic pressures, liquid ambient temperatures and bubble initial radius. An optimal acoustic pressure range and a nucleation temperature threshold as function of bubble radius were determined. The capability of moderate power ultrasound to trigger ice nucleation at low undercooling level and for a wide distribution of bubble sizes has thus been assessed on the theoretical ground.
\end{abstract}

Keywords:

Ultrasound

Cavitation

Single bubble

Liquid undercooling

Ice

Nucleation rate

\section{Introduction}

\subsection{Context and aim}

Controlling the distribution of ice crystal sizes is a key issue in industrial freezing and freeze-drying processes. Ultrasound is already known to be able to initiate the nucleation of ice in undercooled aqueous solutions and to make the freezing reproducible at the desired temperature. But ice crystals' size predictive tools are still missing for design and optimization of ultrasound assisted freezing processes.

As concerns sono-crystallization from solutions, quantitative theoretical prediction of nucleation was pioneered by Virone et al. [1] and applied to ammonium sulphate crystals. However as concerns crystallization from melts and to our best knowledge, Saclier et al. [2] were for the time being the only authors proposing a fully predictive model of ice nucleation triggered by inertial acoustic cavitation. Their model was applied to a $1 \mathrm{ml}$ pure water sample with a known (measured) bubbles' size distribution and the total number of nuclei was calculated as function of the acoustic pressure and water temperature. The model involved however several simplifications and approximations, especially concerning

\footnotetext{
* Corresponding author.

E-mail address: peczalski@lagep.univ-lyon1.fr (R. Peczalski).
}

heat balance of the bubble, heat transfer in the water around the bubble and water thermo-physical properties at very high pressures. The goal of this study was to develop a finer and more comprehensive modeling and to propose nucleation thresholds for bubbles of different initial radii.

The authors of the present study have chosen to continue working with the 'pressure rise effect' [3] (see next section) as the nucleation mechanism and set up a comprehensive theoretical model starting from the bubble wall motion induced by inertial cavitation (see part I) and finishing with the number of nuclei generated by the collapse of a single bubble. In the previous paper [4] (part 1), the pressure and temperature fields close to a bubble undergoing inertial acoustic cavitation were simulated and it was shown that extremely strong liquid water pressures but quite moderate temperatures were reached near the bubble wall just after the collapse.

The next step described in this paper (part 2) was to develop a model of ice nucleation and integrate the nucleation volume rate equation over space and time, using the pressure and temperature profiles induced by the collapse of the bubble in order to obtain finally the number of generated nuclei.

As concerns nucleation kinetics, the classical equation for primary stationary homogeneous nucleation was applied [5], but the parameters of this equation had to be made pressure and temperature dependent. 


\subsection{Bibliographical review}

In this section a short literature review concerning first the experimental evidence of the effect of ultrasound on ice crystallization and second the theories about the ice nucleation mechanism by ultrasound, will be presented.

Inada et al. [6] and Zhang et al. [7] studied the potential application of ultrasonic waves to produce ice slurries. They found out that applying ultrasound greatly increased the probability of phase change from undercooled water to ice at a given initial water temperature. The probability of phase change was calculated as the ratio of the number of successful freezing tests to the total number of tests in given conditions. The occurrence of phase change induced by ultrasound increased with the total number of gas bubble nuclei in undercooled water, independently of other experimental conditions.

Chow and co-authors [8,9] evaluated the effect of ultrasounds on the primary and secondary nucleation of ice in sucrose solutions. They presented direct optical observations of crystals formed around an immersed sonication probe, around a single levitating and cavitating bubble and in a special ultrasonic cold stage. They pointed out that the primary nucleation of ice in sucrose solutions can be achieved at higher nucleation temperatures in the presence of ultrasound. It was also shown that the nucleation temperatures of ice increase with increasing ultrasonic power. It was observed that pre-existing ice crystals could be fragmented by ultrasound, which modifies the size distribution and generates nucleation sites.

Nakagawa et al. [10] and Hottot et al. [11] carried out freezing and freeze-drying experiments with an aqueous solution of mannitol in a small glass tube (vial). The vials were cooled down and sonicated by means of a cold vibrating plate. Ice crystals were observed using reflected-light optical microscopy over frozen samples in transversal and longitudinal sections. It was shown that the nucleation could be readily triggered at selected sample temperature values below the equilibrium freezing temperature and that small and numerous ice crystals were obtained at lower nucleation temperature (higher undercooling level), while large and directional ice crystals (dendrite type) were obtained at a higher nucleation temperature (lower undercooling level). Saclier et al. [12] continued the work on mannitol solution sono-freezing in vials. Using the same apparatus and methodology, they confirmed the above qualitative results but further adopted a second order experimental design and quantitatively assessed the effect of both the nucleation temperature and the acoustic power on the final crystal size and shape. The ice crystal size was found to decrease with both the level of undercooling and the acoustic power level, whereas their circularity was found to increase with these parameters.

Kiani et al. [13] studied ultrasound-assisted nucleation of pure water, sucrose solution, and agar gel samples inserted in tubing vials. The vials were immersed in an ultrasonic bath. Ultrasound was applied continuously for different durations and at different sample temperatures in the range up to $5^{\circ} \mathrm{C}$ below the freezing point. They observed that ultrasound can trigger ice nucleation with high repeatability at the targeted temperatures and hence can be used to control the onset of nucleation. In a continuation of this study [14], the effect of ultrasound intensity on the nucleation of ice in agar gel samples was additionally studied. It was observed that ultrasound irradiation was able to initiate nucleation at different undercooling levels of the gel if optimum intensity and duration of ultrasound application were chosen.

Although ultrasound has long been used to initiate nucleation in undercooled aqueous solutions, the exact mechanism that explains this effect is not yet well known. Acoustic cavitation (the sudden formation and collapse of gas bubbles in liquids by means of ultrasound) appears to cause the nucleation of ice.
There is a distinction between stable cavitation when a bubble pulsates about an equilibrium radius over many acoustic cycles and inertial cavitation when the bubble grows extensively and finally collapses [15].

According to the theoretical study by Hickling [3] very high positive pressures occurring during the final stage of the collapse of a bubble in inertial cavitation increase the equilibrium temperature of water and ice (VI or VII, denser than liquid water), thus enhancing nucleation of a specific ice solid phase. The weak point of this scenario is the uncertainty about a subsequent transformation of the nucleated high pressure ice phase into low pressure regular ice Ih. According to another model [16], nucleation is caused by negative pressures that follow the collapse of the cavitation bubble. This effect will produce low pressure ice Ih, but this idea was questioned by the experiments of Ohsaka and Trinh [17].

On the ground of the Hickling's hypothesis, Inada et al. [6] made an attempt to deduce the probability of phase change from undercooled water to ice from the probability of inertial acoustic cavitation of a given bubble population. But in order to fit their experimental results, they needed to adjust two parameters and their model was not predictive.

According to some other experimental results [9], the moderate oscillation of a bubble in stable cavitation may also induce ice nucleation. These authors suggest micro-streamings as a factor promoting nucleation. The other possible nucleation mechanism is the concentration and agglomeration of ice clusters near the bubble due to pressure diffusion (transfer mechanism driving the densest species toward high-pressure zones). On the basis of theoretical considerations, Grossier and co-workers $[18,19]$ argued that the very high pressure gradients that are needed for pressure diffusion to be effective are attainable only for collapsing bubbles. They thus indirectly refuted stable cavitation as a nucleation initiator.

As suggested by Kordylla et al. [20] and Yasui et al. [21], the occurrence of gas/liquid interface (at the bubble wall) in a supersaturated sonicated solution may induce a kind of heterogeneous nucleation, by reducing interfacial solid-liquid tension and thus reducing the nucleation energy barrier $\left(\Delta G_{\mathrm{c}}\right)$ according to the classical nucleation theory (CNT). Kordylla et al. [20] has in fact identified the contact angle minimizing a least-square error between experimental and theoretical values. This is however rather a 'curve fitting' approach than a real physical model of sono-nucleation. Some studies have demonstrated that single bubbles do not exhibit this effect [8].

More importantly, applying CNT in a global manner for sono-crystallization is not straightforward, since this theory relies on a (metastable) equilibrium hypothesis. At the scale of a bubble, there exists huge pressure gradients (and to a lesser extent temperature gradients) near the bubble, so that the supersaturation itself is distributed over space and time. Thus, considering the nucleation work $\left(\Delta G_{\mathrm{c}}\right)$ as constant is a somewhat rough approximation in this situation. Possibly, one could invoke a local equilibrium hypothesis, calculate a spatio-temporal profile $\Delta G_{\mathrm{c}}(r, t)$ around the bubble, and deduce a local instantaneous nucleation rate. This is the idea underlying the 'pressure effect' tentatively quantified in the literature [1,2] and developed in this study.

However, even when accounting for such refinement, the hypothesis of stationary nucleation is always done. In fact, local supersaturation varies on the same time scale as the bubble radius (or shorter in the case of shock waves). If this time scale is of the same order of magnitude as the so-called nucleation time lag, nucleation may be in fact transient. Thus, estimations based on quasi-stationary nucleation may be largely overestimated [22].

A quite novel idea about the nucleation mechanism has arisen very recently [23]. The physics of inertial cavitation states that the bubble interior heats up due to a quasi-adiabatic compression just before the collapse, but then cools down due to a 
quasi-adiabatic expansion of the bubble just after the collapse. According to simulations, the temperature of the gas mixture inside the bubble falls to between $-30{ }^{\circ} \mathrm{C}$ and $-60{ }^{\circ} \mathrm{C}$ after the collapse, depending on operating conditions. According to the water state diagram, this means that all the water vapor is in a highly metastable undercooled state and could easily transform into ice Ih. This raises two issues, however: is the metastable water period long enough to induce ice nucleation? And how might the ice nuclei invade the surrounding liquid, breaking the bubble wall?

Even if different theories exist and some experimental results were published, no quantitative predictive modeling approach has been proposed until now to assess the impact of ultrasound on nucleation and crystallization of ice in undercooled aqueous solutions, except for the work of Saclier et al. [2].

\section{Modeling}

\subsection{Nucleation kinetics}

Nucleation is the process of random generation of small (nano-metrical) aggregates (nucleus) of the new phase that have the ability for irreversible growth to macroscopically large sizes. The driving force for nucleation is the difference of chemical potential of the considered component in the old (liquid) and the new phase (solid). In the case of crystallization from melt by cooling, this driving force is expressed as a function of the so-called 'undercooling' which is the difference between the actual temperature of the liquid $T$ and the theoretical melting temperature of the solid phase $T_{m}[5]$ :

$\Delta \mu=-L_{m}(P) \frac{T-T_{m}(P)}{T_{m}(P)}$

where $L_{m}$ is the solid phase melting enthalpy per unit mass, $P$ is the liquid phase pressure. Atypically $\Delta \mu$ is expressed here in $\mathrm{J} / \mathrm{kg}$. The subscript ' $m$ ' points out values in solid-liquid thermodynamical equilibrium conditions. The expression above should have been corrected with a term involving the difference between liquid phase and solid phase specific heat capacity $\left(\Delta c_{\mathrm{pm}}\right)$ for very high 'undercoolings' $\left(\Delta T>2 L_{m} /\left(\Delta c_{\mathrm{pm}}\right)\right)$. But due to the important uncertainty about the ice heat capacity values at high pressures, this correction had not been applied. The ice melting enthalpy can be expressed with the slope of the equilibrium temperature curve by the classical Clausius-Clapeyron equation:

$L_{m}(P)=\left(\frac{d T_{m}}{d P}\right)^{-1} T_{m}(P)\left[\frac{1}{\rho_{l m}(P)}-\frac{1}{\rho_{s m}(P)}\right]$

where $L_{m}$ is expressed in $\mathrm{J} / \mathrm{kg}$ and $\rho$ is the mass density of liquid (1) or solid phase (s).

In this study only primary homogeneous nucleation was considered, which is occurring when the clusters of the new phase are in contact only with the old phase and with no other phases or species. According to the classical nucleation theory [5], the rate of formation of such clusters is basically controlled by the energy barrier a cluster has to overcome in its evolution to a stable solid phase and by the frequency of new molecule attachment to the cluster. The energy barrier is calculated as the Gibbs free energy variation necessary for a cluster to reach its critical size (minimum radius of a stable spherical nucleus). The total free energy variation of a cluster is a sum of the free-energy gain due to the liquid-solid phase change and the free-energy loss associated with the creation of the liquid-solid interface. The total free-energy function has a maximum at the critical cluster size. This maximum corresponds to the energy barrier for cluster formation and is inversely proportional to the square of the undercooling of the melt. The Gibb's free enthalpy of one critical size cluster formation writes:
$\Delta G_{c}(T, P)=\frac{16 \pi}{3} \frac{\sigma_{s l}^{3}(T, P)}{\rho_{s}^{2}(T, P) \Delta \mu^{2}(T, P)}$

where $\Delta G_{c}$ is expressed in $\mathrm{J}$ (per cluster) and $\sigma_{\mathrm{sl}}$ is the liquid-solid surface tension.

The primary homogenous nucleation rate $J$ is defined as the number of nuclei generated per unit of volume of the liquid and per unit of time. The nucleation rate represents the frequency of appearance at time $t$ of supercritical clusters per unit of volume. At an absolute temperature $T$, the fraction of clusters that have the size greater than the critical one (by just a single molecule) can be calculated from statistical mechanics and is given by the Boltzmann distribution based on the hypothesis that the probability of some state arising by fluctuation is proportional to the negative exponential of the entropy change involved. The nucleation rate writes then:

$J(T, P)=J_{0}(T, P) \exp \left[-\frac{\Delta G_{c}(T, P)}{k T}\right]$

where $k$ is the Boltzmann constant. It is expressed here in number per $\mathrm{m}^{3}$ and per $\mathrm{s}$.

The pre-exponential factor $J_{0}$ describes the rate of adjoining new molecules to existing critical clusters (aggregation). The diffusion of critical clusters in the liquid is usually assumed as the aggregation rate limiting mechanism. If the diffusion coefficient is expressed by the Stokes-Einstein formula (of spherical particles through a liquid with low Reynolds number), the dynamic viscosity of the liquid $\eta_{1}$ appears as a key parameter. The kinetic factor of aggregation writes then [5]:

$J_{0}(T, P)=\frac{4\left[\sigma_{\mathrm{sl}}(T, P) k T\right]^{1 / 2}\left[\rho_{\mathrm{s}}(T, P) N_{\mathrm{A}}\right]^{5 / 3}}{\eta_{\mathrm{l}}(T, P)(6 M)^{5 / 3} \pi^{1 / 3}} \exp \left[-\frac{L_{m}(P) M}{k N_{\mathrm{A}} T_{m}(P)}\right]$

where $M$ is the molecular mass of the liquid, $N_{\mathrm{A}}$ the Avogadro number.

\subsection{Number of nuclei}

As shown in equations presented above, the nucleation rate is a function of liquid pressure and temperature. By means of the model developed in the part 1 of this study, the pressure and temperature in the liquid adjacent to the bubble wall just before and just after the collapse can be calculated as function of time $t$ and radial distance from bubble center $r$. In this way the nucleation rate $J(P, T)$ becomes a function of radius and time $J(r, t)$ and can be integrated over a liquid volume surrounding the bubble and over time in order to calculate the number of nuclei $N$ generated by the bubble.

$N=4 \pi \iint_{R, t}^{\infty} J(r, t) r^{2} d r d t$

For each time step the integration was started at the bubble wall and was stopped at a distance where no more nuclei were produced $(N<1)$. In the same way the time integration was started at the collapse and stopped when the number of generated nuclei became constant.

Eq. (5) is valid only for quasi-stationary nucleation. The necessary condition for stationary nucleation is that the time scale of driving force variation is greater than the time scale $\tau$ of new phase particle diffusion to the cluster. It can be written as:

$d / d t\left[\frac{\Delta G_{c}(t)}{k T(t)}\right]<\frac{16}{\pi^{3} \tau(t)}$

and

$\tau(t)=\frac{9 N_{\mathrm{A}} \eta_{1}\left(\Delta G_{c}\right)^{4 / 3} \rho_{\mathrm{s}}^{2} L_{m}^{2}}{8 \pi^{2} M \sigma_{\mathrm{sl}}^{3}} \exp \left[-\frac{L_{m} M}{k N_{\mathrm{A}} T_{m}}\right]$ 
As the time available for nucleation after a bubble collapse is extremely short, this condition was verified at every time step of the simulation. The nuclei generated in non-stationary conditions were discarded.

\subsection{Water and ice properties}

In the context of a bubble collapse event inducing very strong pressure and temperature gradients in the neighboring liquid, all the parameters of the equations given above had to be made pressure and temperature dependent. The parameters varying the most with pressure were the equilibrium ice-water temperature (which determined the undercooling level), the ice-water surface energy, the ice melting enthalpy and ice density. The great difficulty was to feed the model with reliable thermo-physical properties of water and ice as literature data are scarce in the very high pressures range involved by the bubble collapse (up to $60 \mathrm{GPa}$ ).

The solid-liquid equilibrium temperature (ice melting temperature) for water was calculated piece by piece for different types of ice compiling data from different sources: Choukroun and Grasset [24] for ice Ih, Wagner et al. [25] for ices III, V, VI and Lin et al. [26] for ice VII. The expression relating the equilibrium temperature to the liquid pressure for all pressure domains writes:

$T_{m}(P)=T_{0}\left(\frac{P-P_{0}}{a}+1\right)^{1 / b}$

and the corresponding coefficients are given in Table 1 . The plot of the above function is given in Fig. 1.

The data concerning the liquid water and ice density were obtained from the work of Choukroun and Grasset [24] for ice Ih, III, V, VI and from the study of Frank et al. [27] for ice VII. Liquid water dynamic viscosity was read in Huber et al. [28] for pressure below $1 \mathrm{GPa}$ and in Abramson [29] for pressure above $1 \mathrm{GPa}$ (with extrapolation above $6 \mathrm{GPa}$ ).

It was finally possible to collect quite reliable pressure dependent data for all properties of liquid water and ice, with the exception of the water solid-liquid surface tension for which a very approximate and not fully validated formula was used. This pressure correction consisted to simply consider the surface tension as proportional to the melting enthalpy. According to Lee and Wang [30] the following expression may be postulated:

$\frac{\sigma_{\text {sli }}^{3}(P, T)}{\rho_{\text {si }}^{2}(P, T)}=\frac{\sigma_{\text {sli }}^{3}\left(P_{0 i}, T\right)}{\rho_{s i}^{2}\left(P_{0 i}, T\right)}\left[\frac{L_{m i}(P)}{L_{m i}\left(P_{0 i}\right)}\right]^{3}$

However, even if one accepts the postulate, this formula should be applied separately for each ice type (represented by the underscript $i, P_{0 i}$ being the lower limit pressure for ice $i$ ). As the starting values for the different ices other than Ih are not available (to our best knowledge), this formula was used all over the entire pressure domain starting from ice Ih. The temperature dependence of ice-water surface tension was accounted for using the correlation of Pruppacher [31]. The surface tension has a huge influence on the nucleation rate since it enters to the third power in an exponential in Eq. (3). The uncertainty about this parameter for high pressures is thus a serious drawback of the proposed model.

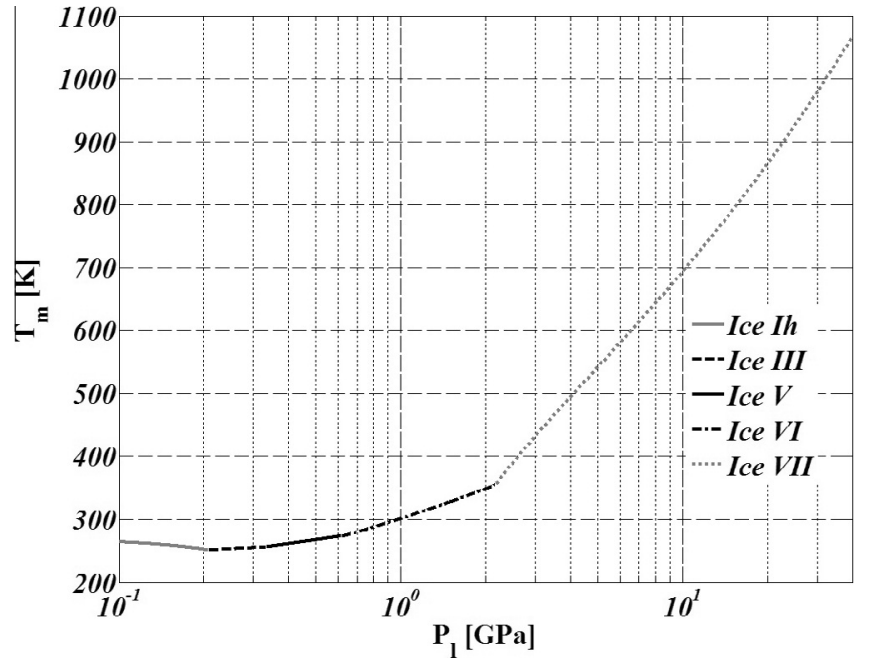

Fig. 1. Water solid-liquid equilibrium (melting) temperature versus pressure. (Data from [24-26]).

\section{Results and discussion}

\subsection{Undercooling}

The 'undercooling', defined as the difference between the liquid temperature and the melting (solid-liquid transition) temperature: $T_{1}-T_{m}$, is the nucleation driving force in a freezing process according to the classical nucleation theory adopted in this paper. The first concern was to determine the undercooling range around the collapsing bubble. The liquid temperature $T_{1}$ profiles near the bubble wall were calculated and shown in part $1[4]$ of this paper. The melting temperature $T_{m}$ profiles were obtained from the liquid pressure profiles (see part 1 [4]) using the water solid-liquid equilibrium curve (see Eq. (9) and Fig. 1). Finally, the $T_{1}-T_{m}$ temperature difference was determined at each radial location around the bubble and for times just following the collapse and was plotted on Fig. 2. When this difference was negative, the liquid was 'undercooled' and the nucleation was possible. To make the undercooling value negative or positive is only matter of convention; in simulation the absolute value was used.

In Fig. 2, the first plotted line (65 ps) corresponds to the time of maximum pressure at the bubble wall, the time zero being the time of the collapse defined as the maximum of bubble wall velocity. As observed on this graph, the collapse induces a huge local undercooling, up to $-370 \mathrm{~K}$ just at the time of maximum pressure, which may trigger nucleation. However, the high undercooling domain (over $-50 \mathrm{~K}$ ) is very limited in time (around $1 \mathrm{~ns}$ after the collapse) and in space (around $2 \mu \mathrm{m}$ from the bubble wall). According to the results obtained in part 1 of this paper, the liquid temperature $T_{1}$ at the bubble wall can reach around $620 \mathrm{~K}$ $\left(R_{0}=5 \mu \mathrm{m}, f_{\mathrm{ac}}=29 \mathrm{kHz}, P_{\mathrm{ac}}=140 \mathrm{kPa}\right)$ with the far field temperature $T_{1 \infty}$ set as $263 \mathrm{~K}$. The melting temperature $T_{m}$ value at the bubble wall can attain around $900 \mathrm{~K}$ because of the liquid pressure $P_{1}$ reaching $23 \mathrm{GPa}$. But the maximum undercooling $(-370 \mathrm{~K})$ is not

Table 1

Coefficients of correlation for water solid-liquid equilibrium temperature (T: K, P: MPa).

\begin{tabular}{|c|c|c|c|c|c|}
\hline Parameter & $\begin{array}{l}\text { Ice Ih [24] } \\
0-209.9 \mathrm{MPa}\end{array}$ & $\begin{array}{l}\text { Ice III [25] } \\
209.9-350.1 \mathrm{MPa}\end{array}$ & $\begin{array}{l}\text { Ice } \mathrm{V}[25] \\
350.1-632.4 \mathrm{MPa}\end{array}$ & $\begin{array}{l}\text { Ice VI [25] } \\
632.4-2170 \mathrm{MPa}\end{array}$ & $\begin{array}{l}\text { Ice VII [26] } \\
2170-22000 \mathrm{MPa}\end{array}$ \\
\hline$P_{0}(\mathrm{MPa})$ & $611.657 \times 10^{-6}$ & 209.5 & 355 & 618.4 & 2170 \\
\hline$T_{0}(\mathrm{~K})$ & 273.15 & 251.15 & 256.43 & 272.73 & 355 \\
\hline$a$ & -414.5 & 101.1 & 373.6 & 661.4 & 850 \\
\hline$b$ & 8.38 & 42.86 & 8.66 & 4.69 & 3.47 \\
\hline
\end{tabular}




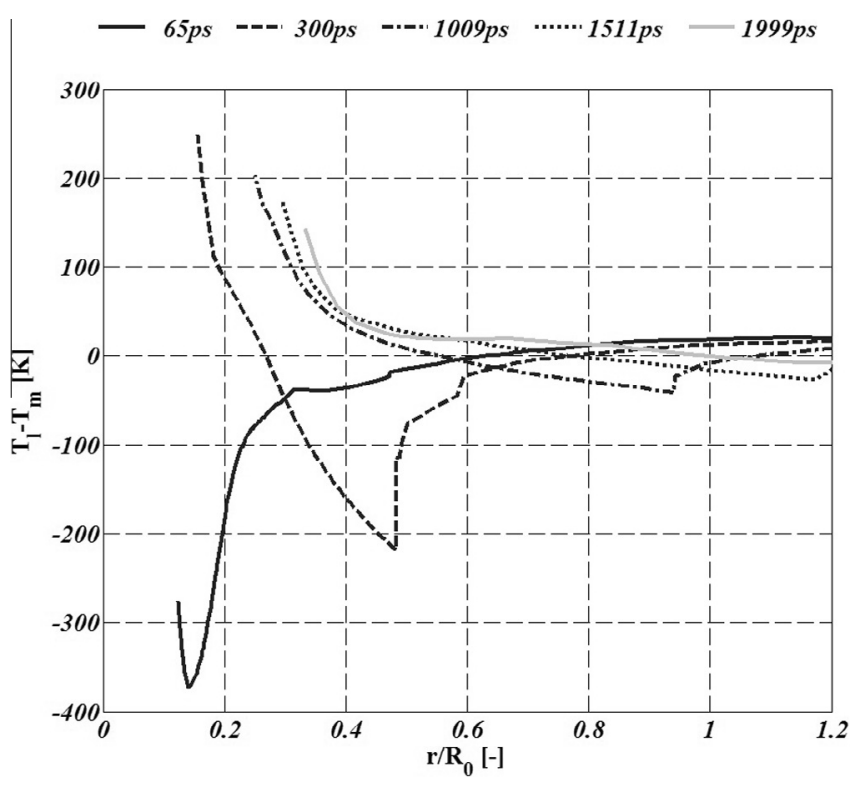

Fig. 2. Liquid undercooling versus the radial position around the bubble and for different times after the collapse $\left(R_{0}=5 \mu \mathrm{m}, f_{\mathrm{ac}}=29 \mathrm{kHz}, P_{\mathrm{ac}}=140 \mathrm{kPa}\right)$.

exactly at the bubble wall, but a little bit away (see Fig. 2) where the pressure is still very high but the $T_{1}$ temperature have already steeply dropped.

\subsection{Nucleation rate}

The volumetric nucleation rate $J$ calculated according to Eqs. (15 ) is plotted as function of liquid pressure and temperature in Fig. 3. This plot is not specific to a collapse event but shows generally how the nucleation rate behaves with changing liquid pressure and temperature in the very high pressures range. For comparison, the $J$ value at $-40{ }^{\circ} \mathrm{C}$ and atmospheric pressure is $30 \mu \mathrm{m}^{-3} \cdot \mathrm{ns}^{-1}$. As shown on Fig. 3 , the nucleation rate $J$ curves have a maximum located around $2.3 \mathrm{GPa}$. The occurrence of this maximum and the significant gradients of $J$ with regard to pressure are due to the contradictory influence of water and ice thermo-physical properties (see also Section 2).
The influence of liquid temperature on the nucleation rate is straightforward. If the temperature is decreased, the increase of the thermodynamic driving force of the phase change (undercooling) is prevailing in spite of the decrease of the values of the kinetic coefficient of the aggregation process. The influence of liquid pressure on the nucleation rate relies on two opposite effects. If pressure is increased, the increasing undercooling, solid density and melting enthalpy tend to increase the nucleation rate. But the influence of these parameters is offset by the substantial increase of the solidliquid surface tension with pressure which results in a decrease of $J$ for pressures above $2.3 \mathrm{GPa}$. Indeed, the surface tension is proportional to the melting enthalpy which depends on the equilibrium temperature curve and density curve and strongly increases with pressure especially for ice VII. There is in particular a step change of the value of ice density and of the slope of the equilibrium curve (see Fig. 1) because of the transition from ice VI to ice VII.

\subsection{Nuclei number}

The integration of $J$ over the time elapsed after collapse and over space around the bubble (see Eq. (6)) gives the number of nuclei produced by a single collapse event. The results are presented first as a plot of isopleths of the nuclei number (in the pressure-temperature coordinates) generated by an air bubble with an initial radius of $5 \mu \mathrm{m}$ driven by an ultrasonic sinusoidal wave at the frequency of $29 \mathrm{kHz}$ (see Fig. 4). The practical integration limits for this case were roughly from 1 to $5 \mu \mathrm{m}$ for radius and from 0 to $2 \mathrm{~ns}$ for time.

According to our results, the nucleation could be triggered starting from an undercooling as low as $5 \mathrm{~K}$ for bubbles of initial radius of $5 \mu \mathrm{m}$ (or around $4 \mathrm{~K}$ for $8 \mu \mathrm{m}$ bubbles, see Fig. 5) if an acoustic pressure amplitude of around $220 \mathrm{kPa}$ is applied. For moderate acoustic pressures up to $220 \mathrm{kPa}$, the number of generated nuclei was found to increase with the undercooling level and with the acoustic pressure, which was in good qualitative agreement with already published experimental results $[6,7,9]$. However, for acoustic pressures above $220 \mathrm{kPa}$ and for a given liquid super-cooling level, the number of nuclei was found not to vary any more with pressure. There is thus a range of acoustic pressure where the ultrasound is the most efficient and increasing the pressures beyond is not useful.

The difficulty in quantitative comparison of the present data and those from literature comes from the different methods used to measure the acoustic power (often confused with the electrical

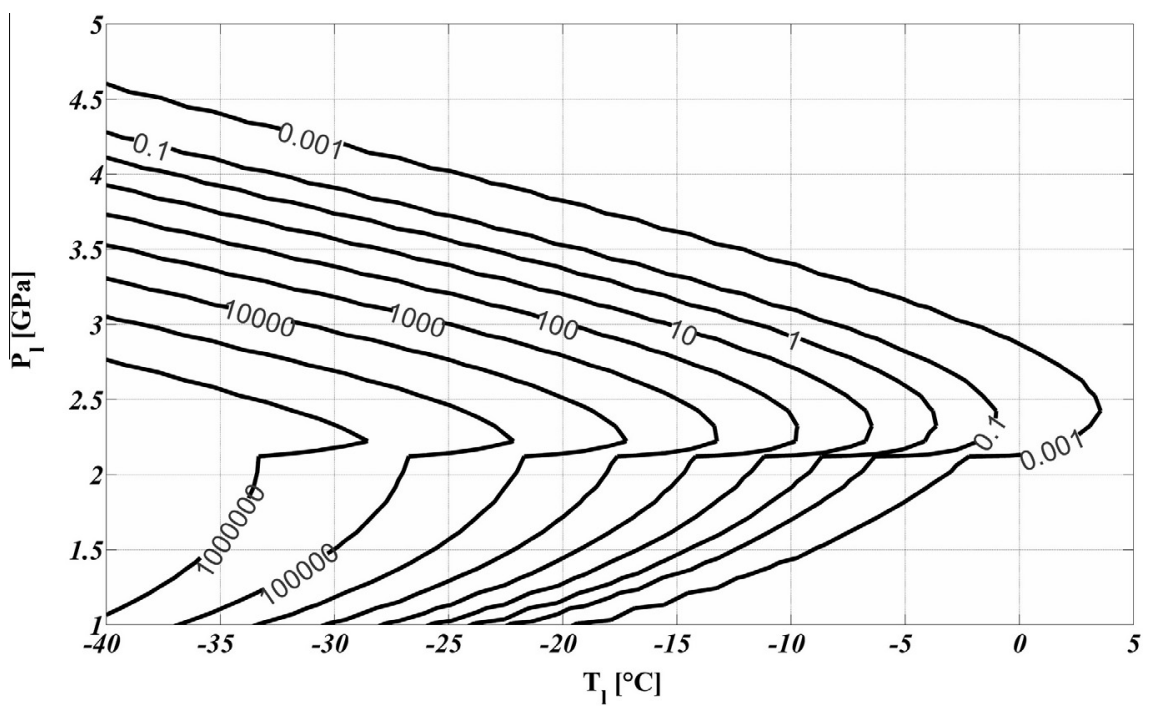

Fig. 3. Nucleation rate per unit volume $\left[\mu \mathrm{m}^{-3} \cdot \mathrm{ns}^{-1}\right]$ versus liquid temperature and pressure. 


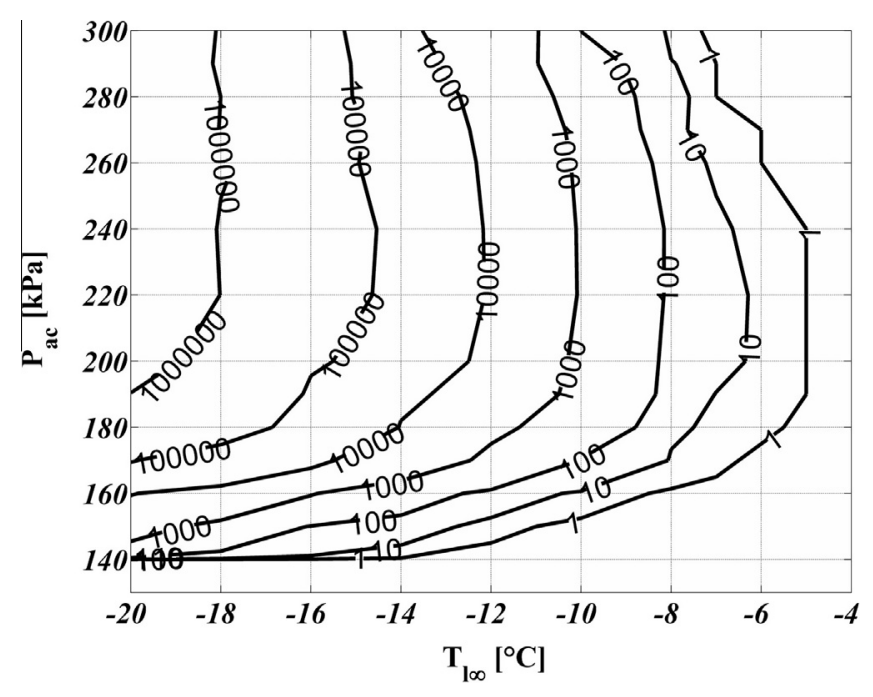

Fig. 4. Number of nuclei as function of initial liquid temperature and acoustic wave amplitude.

power delivered to the transducer) and different output parameters (nucleation probability or nucleation temperature) used to evaluate the nucleation rate (see Section 1). For instance, for the same operating conditions, the nucleation probability of $100 \%$ according to Inada et al. [6] corresponds roughly to a nuclei number of 10 , according to our results.

Moreover, if it is supposed that the greater the number of nuclei, the smaller the crystal size, then the trends predicted by the theoretical model described above corresponded quite well, qualitatively speaking, to those given by an empirical model based on an experimental design proposed previously by Saclier et al. [12] (see preceding paragraph). A quantitative validation of the theoretical model was not possible, however, because the responses of the experimental design and of the simulations were not directly comparable. The former provided a number of ice nuclei in initial undercooled liquid, and the latter the size of ice crystals in the final frozen solid. A necessary continuation of this work will be therefore the further reconciliation of experimental and model predicted data.

Finally, the ice nucleation temperature threshold is given as function of acoustic pressure and initial bubble radius at the frequency of $29 \mathrm{kHz}$. The contour curves corresponding to several initial liquid temperatures and representing the conditions for

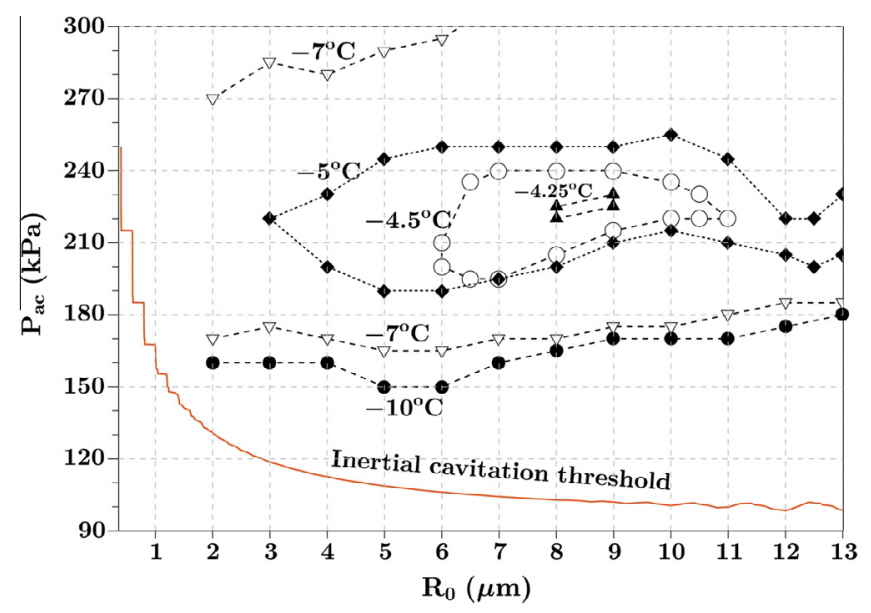

Fig. 5. Isotherms of nucleation threshold as function of initial bubble radius and acoustic pressure. generation of at least one nucleus are plotted on Fig. 5, along with the line corresponding to inertial cavitation threshold. The latter was obtained by using the dynamic equation of bubble wall motion (part 1, [4]) and the cavitation criterion of bubble radius expanding at least twice its initial value. Each contour curve has an upper and lower branch and the zones of possible nucleation lie within these branches. This figure shows even more clearly the existence of an optimal pressure range centered on $225 \mathrm{kPa}$, especially for low undercooling. There is also an optimal bubble size range centered on $8.5 \mu \mathrm{m}$. Nucleation can be already achieved at $-5{ }^{\circ} \mathrm{C}$ over a large size domain (radius from 3 to $13 \mu \mathrm{m}$ ) providing the appropriate acoustic pressure. The influence of the initial radius on nucleation threshold reflects the nonlinear evolution of the bubble dynamics with the initial radius. Even if it is not as critical as the applied acoustic pressure, the control of initial bubble size may be helpful for achieving nucleation at a desired temperature.

\section{Conclusion}

In the preceding paper (part 1), a theoretical model of acoustic inertial cavitation was developed on the ground of a literature survey. The pressure and temperature fields close to a bubble undergoing inertial cavitation were simulated and it was shown that strong liquid water undercooling was reached locally just after the collapse.

Starting from the Hickling's 'pressure rise effect' hypothesis, the goal of this study was to evaluate rigorously the number of ice nuclei generated by the collapse of a single gas bubble driven by an ultrasonic wave in initially undercooled water (temperature below $0{ }^{\circ} \mathrm{C}$ ). First, the previous model was extended to incorporate the pressure and temperature dependent kinetic model of primary homogeneous ice nucleation. Second, an integration of the nucleation rate over the liquid volume around the bubble and over time after the collapse was performed in order to get the number of nuclei. The calculations were performed for different driving acoustic pressures, ambient liquid temperatures and bubble initial radius.

It was proved that nucleation could be triggered at low undercooling levels and for a wide distribution of bubble sizes, as far as the applied acoustic pressure lies in a specific appropriate range. Nucleation was found to occur at a higher liquid temperature for larger bubbles up to a certain bubble size. Thus the control of nucleis (crystals) number would be facilitated by the control of the bubble population which might be achieved by saturating the liquid sample with air or by pre-sonicating the sample with ultrasound of adapted frequency and amplitude. As the number of nuclei determines the size of crystals for a given liquid volume, the model developed in parts 1 and 2 allows for predicting trends of ice crystals size variation as function of operating conditions. It provides guidance for using ultrasound to control ice crystals size in frozen products and thus for optimal design of industrial freezing processes.

\section{Acknowledgements}

This work was financially supported by the French governmental research funding agency ANR in the frame of the project '09-BLAN-0040-SONONUCLICE'.

\section{References}

[1] C. Virone, H.J.M. Kramer, G.M. van Rosmalen, A.H. Stoop, T.W. Bakker, Primary nucleation induced by ultrasonic cavitation, J. Cryst. Growth 294 (1) (2006) 9-15.

[2] M. Saclier, R. Peczalski, J. Andrieu, A theoretical model for ice primary nucleation induced by acoustic cavitation, Ultrason. Sonochem. 17 (2010) 98-105. 
[3] R. Hickling, Transient, high pressure solidification associated with cavitation in water, Phys. Rev. Lett. 73 (21) (1994) 2853-2856.

[4] C. Cogné, S. Labouret, R. Peczalski, O. Louisnard, F. Baillon, F. Espitalier, Theoretical model of ice nucleation induced by acoustic cavitation. Part 1 : Pressure and temperature profiles around a single bubble, Ultrason. Sonochem. (2015), http://dx.doi.org/10.1016/j.ultsonch.2015.05.038.

[5] D. Kashchiev, Nucleation: Basic Theory with Applications, ButterworthHeinemann, Boston, 2000.

[6] T. Inada, X. Zhang, A. Yabe, Y. Kozawa, Active control of phase change from supercooled water to ice by ultrasonic vibration 1. Control of freezing temperature, Int. J. Heat Mass Transfer 44 (2001) 4523-4531.

[7] X. Zhang, T. Inada, A. Yabe, S. Lu, Y. Kozawa, Active control of phase change from supercooled water to ice by ultrasonic vibration 2. Generation of ice slurries and effect of bubble nuclei, Int. J. Heat Mass Transfer 44 (2001) 4533-4539.

[8] R. Chow, R. Blindt, R. Chivers, M. Povey, The sonocrystallisation of ice in sucrose solutions: primary and secondary nucleation, Ultrasonics 41 (8) (2003) 595-604.

[9] R. Chow, R. Blindt, R. Chivers, M. Povey, A study on the primary and secondary nucleation of ice by power ultrasound, Ultrasonics 43 (4) (2005) 227-230.

[10] K. Nakagawa, A. Hottot, S. Vessot, J. Andrieu, Influence of controlled nucleation by ultrasounds on ice morphology of frozen formulations for pharmaceutical proteins freeze-drying, Chem. Eng. Process. 45 (9) (2006) 783-791.

[11] A. Hottot, K. Nakagawa, J. Andrieu, Effect of ultrasound-controlled nucleation on structural and morphological properties of freeze-dried mannitol solutions, Chem. Eng. Res. Des. 86 (2) (2008) 193-200.

[12] M. Saclier, R. Peczalski, J. Andrieu, Effect of ultrasonically induced nucleation on ice crystals' size and shape during freezing in vials, Chem. Eng. Sci. 65 (10) (2010) 3064-3071.

[13] H. Kiani, Z. Zhang, A. Delgado, D. Sun, Ultrasound assisted nucleation of some liquid and solid model foods during freezing, Food Res. Int. 44 (9) (2011) 2915-2921.

[14] H. Kiani, D. Sun, A. Delgado, Z. Zhang, Investigation of the effect of power ultrasound on the nucleation of water during freezing of agar gel samples in tubing vials, Ultrason. Sonochem. 19 (3) (2012) 576-581.

[15] R.T. Knapp, J.W. Daily, F.G. Hammitt, Cavitation, McGraw-Hill, New York, 1970.

[16] J.D. Hunt, K.A. Jackson, Nucleation of solid in an undercooled liquid by cavitation, J. Appl. Phys. 37 (1) (1966) 254-257.
[17] K. Ohsaka, E.H. Trinh, Dynamic nucleation of ice induced by a single stable cavitation bubble, Appl. Phys. Lett. 73 (1) (1998) 129-131.

[18] R. Grossier, O. Louisnard, Y. Vargas, Mixture segregation by an inertial cavitation bubble, Ultrason. Sonochem. 14 (4) (2007) 431-437.

[19] O. Louisnard, F.J. Gomez, R. Grossier, Segregation of a liquid mixture by a radially oscillating bubble, J. Fluid Mech. 577 (2007) 385-415.

[20] A. Kordylla, T. Krawczyk, F. Tumakaka, G. Schembecker, Modeling ultrasound induced nucleation during cooling crystallization, Chem. Eng. Sci. 64 (8) (2009) $1635-1642$.

[21] K. Yasui, T. Tuziuti, K. Kato, Numerical simulations of sonochemical production of $\mathrm{BaTiO}_{3}$ nanoparticles, Ultrason. Sonochem. 18 (5) (2011) 1211-1217.

[22] G. Shi, J.H. Seinfeld, K. Okuyama, Transient kinetics of nucleation, Phys. Rev. A 41 (4) (1990) 2101.

[23] W. Montes-Quiroz, F. Baillon, J.J. Letourneau, F. Espitalier, O. Louisnard, Freezing conditions in inertial cavitation bubbles during post-collapses expansions, in: Proceedings of the 13th Meeting of the European Society of Sonochemistry ESS13, Lviv Ukraine, OC-48, 2012.

[24] M. Choukroun, O. Grasset, Thermodynamic model for water and high-pressure ices up to $2.2 \mathrm{GPa}$ and down to the metastable domain, J. Chem. Phys. 127 (2007) 124506

[25] W. Wagner, A. Saul, A. Pruss, International equations for the pressure along the melting and along the sublimation curve of ordinary water substance, J. Phys. Chem. Ref. Data 23 (3) (1994) 515-527.

[26] J.-F. Lin, B. Militzer, V.V. Struzhkin, E. Gregoryanz, R.J. Hemley, H.-K. Mao, High pressure-temperature Raman measurements of $\mathrm{H}_{2} \mathrm{O}$ melting to $22 \mathrm{GPa}$ and 900 K, J. Chem. Phys. 121 (17) (2004) 8423-8427.

[27] M.R. Frank, Y. Fei, J. Hu, Constraining the equation of state of fluid $\mathrm{H}_{2} \mathrm{O}$ to 80 GPa using the melting curve, bulk modulus, and thermal expansivity of ice VII, Geochim. Cosmochim. Acta 68 (13) (2004) 2781-2790.

[28] M.L. Huber, R.A. Perkins, A. Laesecke, D.G. Friend, J.V. Sengers, M.J. Assael, I.N. Metaxa, E. Vogel, R. Mares, K. Miyagawa, New international formulation for the viscosity of $\mathrm{H}_{2} \mathrm{O}$, J. Phys. Chem. Ref. Data 38 (2) (2009) 101-125.

[29] E.H. Abramson, Viscosity of water measured to pressure of $6 \mathrm{GPa}$ and temperatures of $300{ }^{\circ} \mathrm{C}$, Phys. Rev. E 76 (2007) 051203.

[30] C.P. Lee, T.G. Wang, The effects of pressure on the nucleation rate of an undercooled liquid, J. Appl. Phys. 71 (11) (1992) 5721-5723.

[31] H.R. Pruppacher, A new look at homogeneous ice nucleation in supercooled water drops, J. Atmos. Sci. 52 (1994) 1924-1933. 